\title{
Adenocarcinoma polimorfo de baixo grau: Relato de caso atípico na região retromolar inferior do paciente jovem
}

\author{
Low-grade polymorphic adenocarcinoma: Atypical case report in the lower retromolar region of \\ the young patient \\ Adenocarcinoma polimórfico de bajo grado: Reporte de caso atípico en región retromolar inferior \\ de paciente joven
}

Recebido: 20/07/2021 | Revisado: 29/07/2021 | Aceito: 31/07/2021 | Publicado: 03/08/2021

\author{
Larissa Rosa Santana Rodrigues \\ ORCID: https://orcid.org/0000-0003-0725-3337 \\ Universidade Paulista, Brasil \\ E-mail: drlarissarosa@hotmail.com \\ Claudio Maranhão Pereira \\ ORCID: https://orcid.org/0000-0001-5511-0387 \\ Universidade Paulista, Brasil \\ E-mail: claudiomaranhao@hotmail.com
}

\begin{abstract}
Resumo
O adenocarcinoma polimorfo de baixo grau (APBG) é uma neoplasia maligna rara em glândula salivar menor. Apresenta prevalência em indivíduos do gênero feminino entre a sexta e a sétima década de vida. Normalmente, inicia-se de forma assintomática, com crescimento lento e baixa agressividade, sendo o palato o local mais frequente. O carcinoma adenóide cístico e o adenoma pleomórfico são as lesões mais frequentes que fazem diagnóstico diferencial com esta neoplasia. A terapia inicial deve consistir em excisão cirúrgica completa, com possibilidade de associação ao tratamento radioterápico, sendo seu prognóstico favorável dependendo da época do diagnóstico. O presente trabalho tem como objetivo relatar um caso clínico de adenocarcinoma polimorfo de baixo grau em uma paciente do gênero feminino, 34 anos de idade, na região de rebordo alveolar inferior esquerdo. Relatos de APBG em rebordo alveolar inferior posterior e em pacientes na $3^{\text {a }}$ década de vida são extremamente escassos na literatura especializada. É de suma importância o conhecimento pelo cirurgião-dentista sobre as malignidades que podem acometer a cavidade bucal. O diagnóstico correto e o estabelecimento de uma conduta clínica ideal são fundamentais para a expectativa de vida e o bom prognóstico para o paciente.
\end{abstract}

Palavras-chave: Adenocarcinoma; Glândulas salivares; Adenoma.

\begin{abstract}
Low-grade polymorph adenocarcinoma (APBG) is a rare malignant neoplasm in the minor salivary gland. It is prevalent in females between the sixth and seventh decade of life. It usually starts asymptomatically, with slow growth and low aggressiveness, and the palate is the most frequent site. Cystic adenoid carcinoma and pleomorphic adenoma are the most frequent lesions that make a differential diagnosis with this neoplasm. Initial therapy should consist of complete surgical excision, with the possibility of association with radiotherapy treatment, with a favorable prognosis depending on the time of diagnosis. This paper aims to report a clinical case of low-grade polymorph adenocarcinoma in a 34-year-old female patient in the lower left alveolar ridge region. Reports of APBG in the posterior inferior alveolar ridge and in patients in the 3rd decade of life are extremely scarce in the specialized literature. It is extremely important for the dentist to know about the malignancies that can affect the oral cavity. Correct diagnosis and the establishment of an ideal clinical approach are essential for the patient's life expectancy and good prognosis.
\end{abstract}

Keywords: Adenocarcinoma; Salivary glands; Adenoma.

\section{Resumen}

El adenocarcinoma polimorfo de bajo grado (APBG) es una neoplasia maligna poco frecuente en la glándula salival menor. Es frecuente en mujeres entre la sexta y séptima década de la vida. Suele iniciarse de forma asintomática, de crecimiento lento y baja agresividad, siendo el paladar el sitio más frecuente, siendo el carcinoma adenoide quístico y el adenoma pleomórfico las lesiones más frecuentes que hacen un diagnóstico diferencial con esta neoplasia. La terapia inicial debe consistir en la exéresis quirúrgica completa, con posibilidad de asociación a tratamiento radioterápico, con pronóstico favorable según el momento del diagnóstico. Este artículo tiene como objetivo reportar un caso clínico de adenocarcinoma polimorfo de bajo grado en una paciente de 34 años en la región del reborde alveolar inferior izquierdo. Los reportes de APBG en la cresta alveolar posterior inferior y en pacientes en la $3^{\mathrm{a}}$ 
década de la vida son extremadamente escasos en la literatura especializada. Es extremadamente importante que el dentista conozca las neoplasias que pueden afectar la cavidad bucal. El diagnóstico correcto y el establecimiento de un abordaje clínico ideal son fundamentales para la esperanza de vida y el buen pronóstico del paciente.

Palabras clave: Adenocarcinoma; Glándulas salivales; Adenoma.

\section{Introdução}

As glândulas salivares são glândulas exócrinas produtoras de secreções fluídas com funções de lubrificação, digestão e proteção do sistema digestório. São divididas em glândulas salivares maiores (parótidas, submandibulares, sublinguais) e glândulas salivares menores, sendo estes presentes na cavidade bucal em maior quantidade. Estes órgãos podem ser acometidos por diversas afecções, incluindo as neoplásicas (Abu et al., 2011; Araujo et al., 2013; Barnes et al., 2005).

As neoplasias de glândulas salivares formam um grupo raro de tumores, cerca de 3 à $5 \%$ de todos os tumores de cabeça e pescoço e entre 0,28 à $1 \%$ dos tumores de toda topografia corporal. Os tumores benignos são os mais frequentes, correspondendo de 54 à 79\% destas doenças, enquanto os malignos representam de 21 à $46 \%$ dos tumor (Baroni et al., 2008) (Buchner et al., 2007; B. W. et al, 2009; Neville et al, 2008).

O adenocarcinoma polimorfo de baixo grau (APBG) em glândula salivar menor é uma neoplasia maligna de baixa frequência, que foi relatada pela primeira vez em 1983. Dos tumores de glândulas salivares, 10 à $15 \%$ são de glândulas salivares menores, sendo que o APBG representa aproximadamente $9 \%$ de todas as neoplasias de glândulas salivares menores. É uma lesão assintomática, de crescimento lento e de baixa agressividade que pode estar associada à dor e à ulceração. Apresenta baixo potencial para metástases, entretanto, há possibilidade de recidivas após períodos prolongados (Carboni et al., 2011; Fava et al., 2001; Fife et al., 2013).

Constata-se uma prevalência em indivíduos do sexo feminino, com faixa etária entre a sexta e a sétima décadas de vida, e o palato é a sua localização mais frequente. O exame histológico mostra uma diversidade morfológica e uma uniformidade citológica, tornando assim difícil seu diagnóstico. O carcinoma adenóide cístico e o adenoma pleomórfico são as lesões mais frequentes que fazem diagnóstico diferencial com esta neoplasia, e a distinção desta lesão para outras neoplasias é importante por seu prognóstico e conduta terapêutica serem diferentes. A terapia inicial deve consistir em excisão cirúrgica completa, com possibilidade de associação ao tratamento radioterápico, sendo seu prognóstico bastante favorável (Já et al., 2008; Jones et al., 2008; JT et al., 1999).

Portanto, fica claro a necessidade do conhecimento por parte do cirurgião dentista sobre as neoplasias malignas para o correto diagnóstico e encaminhamento do paciente a um centro de tratamento especializado. O presente trabalho objetiva relatar um caso clínico de APBG em paciente do sexo feminino, 34 anos de idade, na região de rebordo alveolar inferior esquerdo, ressaltando a importância do diagnóstico diferencial desta com outras neoplasias de glândulas salivares que apresentam comportamento biológico e prognóstico distintos.

\section{Metodologia}

Este artigo trata- se de um relato de caso clínico, pois aborda de forma descritiva e qualitativa um caso de adenocarcinoma em uma paciente jovem (Pereira et al., 2018). O presente estudo relata um caso clínico de adenocarcinoma polimorfo de baixo grau em uma paciente jovem. Todas as informações foram obtidas através do prontuário da paciente, de exames de imagem e laudo histopatológico. A paciente concordou com a pesquisa e autorizou assinando o do Termo de Consentimento Livre e Esclarecido (TCLE). 


\section{Relato do Caso}

Paciente com 34 anos de idade, gênero feminino, foi encaminhada para clínica de odontologia da Universidade Paulista para avaliação de “caroço na boca". Segundo a paciente, a alteração surgira há cerca de meses e era assintomática, todavia estava crescendo gradativamente. Ainda durante anamnese, a paciente relatou não ter traumatizado a região no momento do surgimento da alteração, e que a mesma não atrapalhava na realização das funções estomatognáticas. Quanto aos antecedentes familiares e a história médica pregressa não houve informações pertinentes ao caso.

Em exame físico extra-oral não foi encontrada nenhuma alteração, entretanto em exame intra-oral foi possível observar aumento de volume nodular, coloração rósea, circunscrito, de aspecto cístico, em rebordo alveolar inferior esquerdo, posterior ao segundo molar, aparentemente extra-ósseo, com cerca de seis centímetros em seu maior diâmetro (Figura 1). Inicialmente, foi solicitada radiografia panorâmica, onde foi possível constatar que o osso mandibular apresentava aspecto de normalidade, contudo, era possível visualizar ligeira reabsorção óssea subjacente a lesão (Figura 2). Em seguida, optou-se por realizar uma punção aspirativa para pesquisa de conteúdo cístico, a qual foi positiva para líquido cístico amarela claro (Figura3). Com base nestes dados clínicos e radiográficos, postulou-se a hipótese diagnóstica de um cisto ou tumor cístico odontogênico extra-ósseo.

Figura 1: Aspecto clínico inicial. Nota-se lesão em região de rebordo alveolar inferior esquerdo.

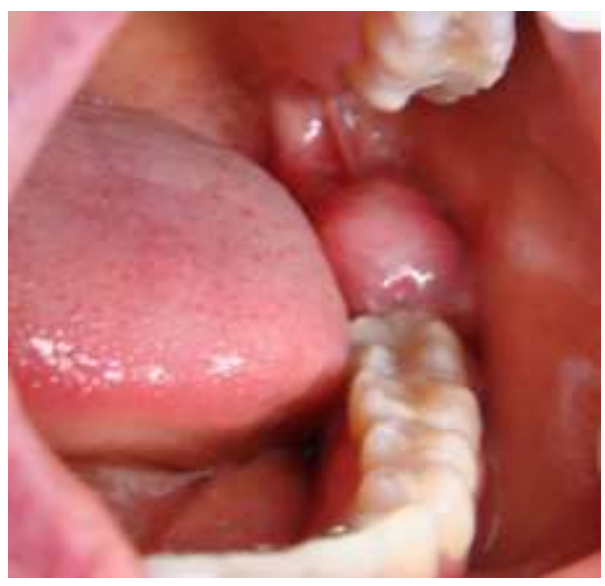

Fonte: Autores.

Figura 2: Panorâmica inicial. Nota-se o não envolvimento de estruturas ósseas.

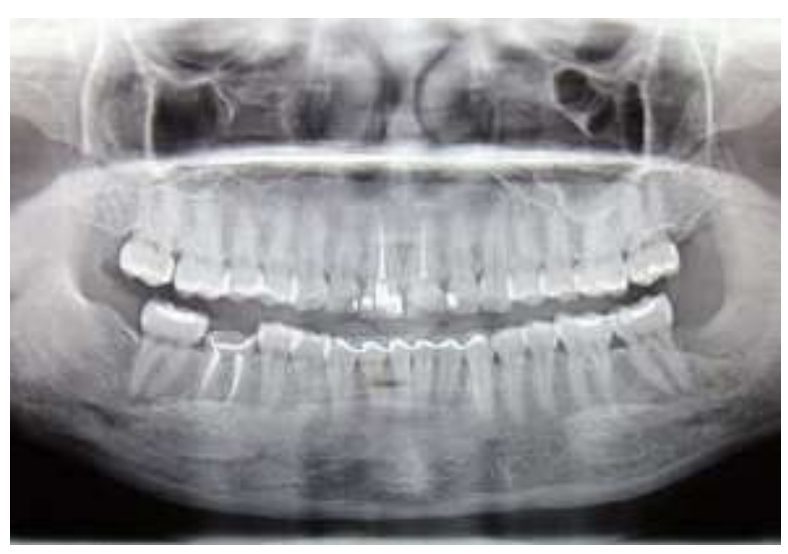

Fonte: Autores. 
Figura 3: Punção aspirativa positiva para líquido (amarelado) cístico.

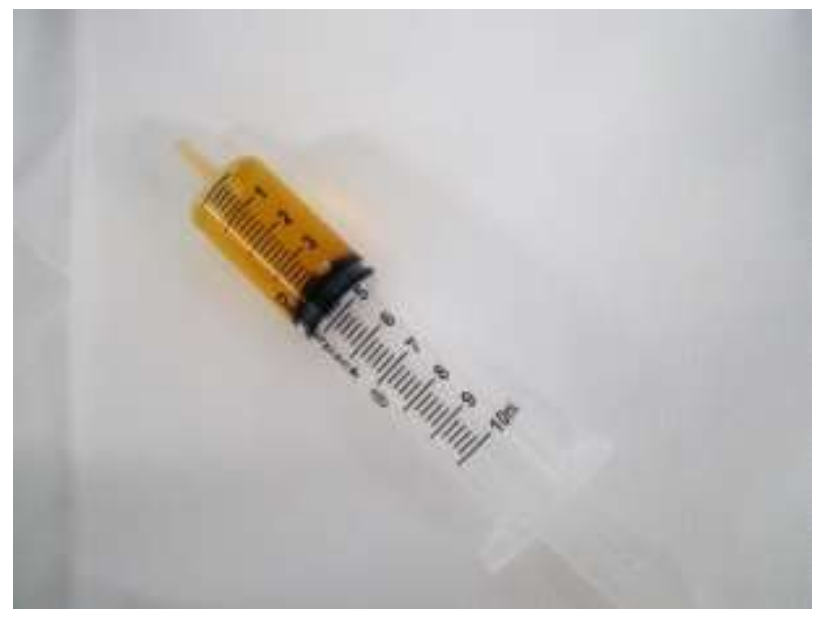

Fonte: Autores.

Posteriormente, a paciente foi submetida a uma biópsia incisional. Durante o procedimento observou-se lesão cística com conteúdo amarelado de fácil divulsão, infiltrando para orofaringe e região de glândula parótida. Após remoção da porção anterior cística notou-se aspecto necrótico e infiltrativo para região posterior. Microscopicamente, foi possível observar uma lesão de padrão sólido-cístico composta por tecido epitelial em arranjo sólido, cordonal e cribriforme, com padrão de crescimento expansivo e infiltrativo. As células tinham forma basalóides com núcleos redondos, cromatina delicada, nucléolos conspícuos, citoplasma pálido, e presença de estroma mixóide (Figura 4). Foi possível ainda observar glândulas salivares menores e tecido linfóide reacional na parede. Com base nos achados histopatológicos, foi estabelecido o diagnóstico de adenocarcinoma polimorfo de baixo grau em glândula salivar menor.

Figura 4: Foi possível observar uma lesão de padrão sólido-cístico composta por tecido epitelial em um arranjo sólido, cordonal ou arranjo, com padrão de crescimento expansivo e infiltrativo.

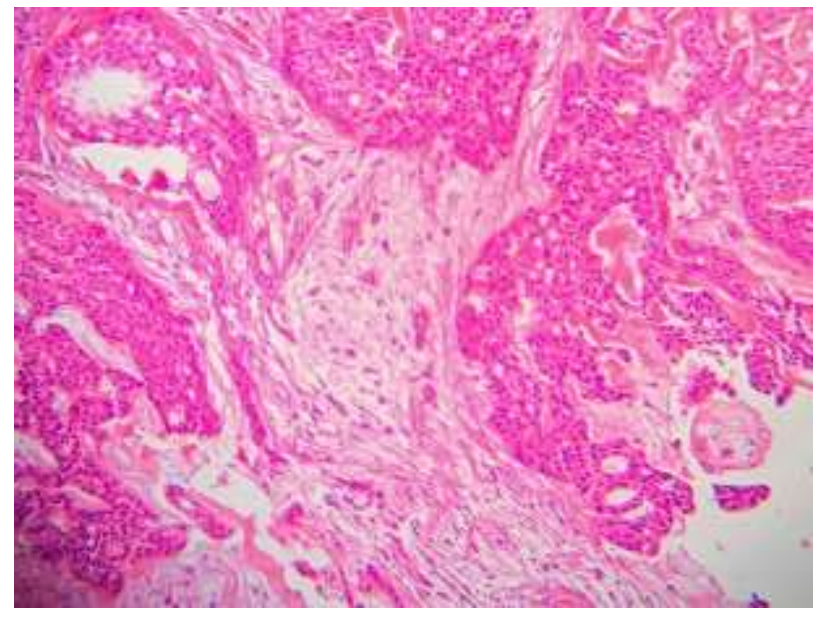

Fonte: Autores.

Logo em seguida, a paciente foi encaminhada ao oncologista para avaliação e terapêutica. Após avaliação especializada foi optado pela ressecção cirúrgica do tumor, a princípio sem radioterapia coadjuvante. Quatro meses após a remoção cirúrgica a paciente retornou com a região sem alterações clínicas evidentes, mas aspecto fibrosado em decorrência da cirurgia. Foi feita radiografia panorâmica de acompanhamento, e também foi possível constatar que a região apresentava-se 
sem a presença de alterações pertinentes (Figuras 5 e 6). Entretanto, no retorno previsto no especialista a paciente não compareceu.

Figura 5: Aspecto clínico quatro meses após a excisão cirúrgica completa.

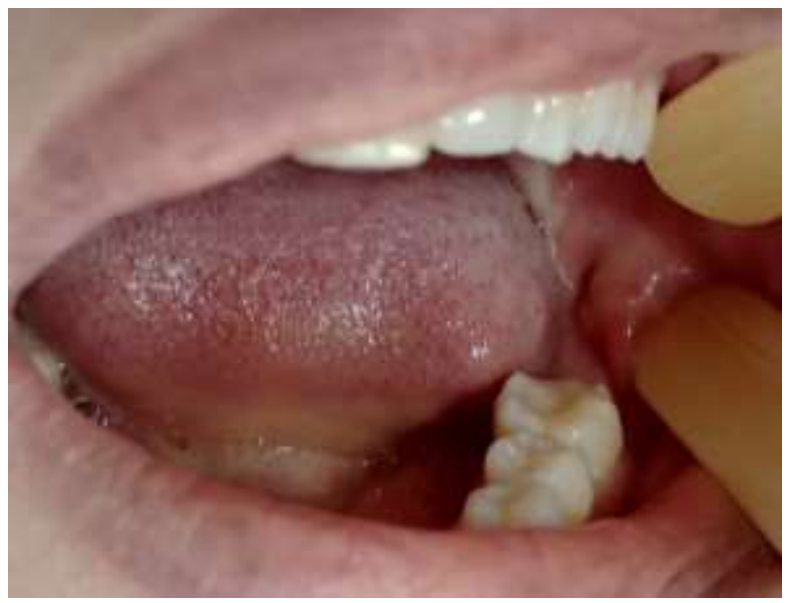

Fonte: Autores.

Figura 6: Panorâmica realizada quatro meses após excisão cirúrgica completa.

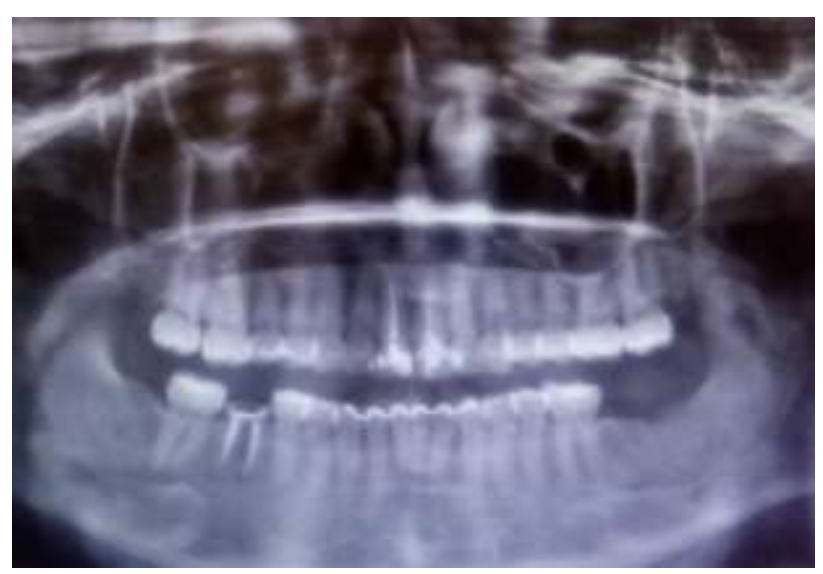

Fonte: Autores.

\section{Discussão}

O APBG é uma neoplasia maligna de glândulas salivares, cujo pico de incidência se da entre a sexta e a sétima década de vida, com uma predileção pelo sexo feminino, sendo a localização mais recorrente a região de palato. Entretanto, neste caso em específico a região acometida foi a de rebordo alveolar inferior esquerdo, sendo assim extremamente raro, e em uma paciente considerada jovem para a taxa de incidência deste tumor (Lima et al., 2005; Ma et al., 2009; Toida et al., 2005).

Em relação às características clínicas, o APBG se apresenta como nódulo de 1 à 4 centímetros e por vezes ulcerados. Neste caso, não houve áreas de ulceração, uma vez que este nódulo possuía superfície lisa, e o diâmetro da lesão $(6 \mathrm{~cm}) \mathrm{se}$ revelou superior ao descrito na literatura para o APBG, o que pode estar relacionado à demora do paciente em procurar ao atendimento especializado, aliado à ausência de sintomatologia dolorosa (Loiola et al., 2009; Mc et al., 2009).

Corriqueiramente, a coloração da superfície do nódulo pode ser castanho-amarelado, e ainda estar associado a sangramentos. Neste caso clínico a lesão era assintomática, de coloração rósea, indo de encontro a maioria da literatura pesquisada (M. C. et al., 2000; O’ rourke et al., 2006). 
Histopatologicamente o APBG apresenta uma grande similaridade com o carcinoma adenóide cístico e o adenoma pleomórfico, cujos tratamentos e prognósticos são distintos. Contudo, as células no carcinoma adenóide cístico apresentam núcleos condensados hipercromáticos, enquanto o APBG possui núcleos palidamente corados. Contrastando com adenoma pleomórfico, o APBG não demonstra evidência de diferenciação condromixóide. Por outro lado, no adenoma pleomórfico, não existe evidência de invasão nos tecidos circundantes. Em biópsias excisionais é importante analisar a periferia do tumor, buscando infiltrações em glândulas salivares e nos tecidos normais circunvizinhos, para auxiliar no estabelecimento do diagnóstico correto do APBG (Milsa et al., 2010; Ms et al., 2006; Kumar et al., 2011).

É essencial observar morfologicamente e histologicamente as neoplasias de glândulas salivares para um correto diagnóstico. O padrão citológico uniforme associado à variedade de padrões histológicos, são corriqueiramente suficientes para o diagnóstico do APBG segundo De Araújo et al (2013).

Neste caso os aspectos histomorfológicos diagnosticaram o APBG. É de suma importância diferenciar o APBG de outras neoplasias das glândulas salivares, em especial das malignas, pois esta é menos agressiva, tendo melhor prognóstico. O tratamento de primeira escolha é a excisão cirúrgica completa com margens livres. Entretanto, dependendo dos limites da lesão e do envolvimento de estruturas nobres, pode ser indicada a cirurgia associada à radioterapia, ou apenas a radioterapia. No caso relatado, a paciente foi encaminhada para o serviço de oncologia do hospital de referência para planejamento cirúrgico e tratamento de suporte, e posteriormente, foi realizada a excisão cirúrgica total da lesão sem associação à radioterapia (Sheetala et al., 2010; Soames et al., 2008). (fonte: TNR 10 - justificado - espaço 1,5).

\section{Conclusão}

O APBG é uma neoplasia maligna típica de glândulas salivares de baixa agressividade, normalmente assintomática. A ausência de sintomatologia dolorosa faz com que os pacientes demorem a procurar atendimento, como o ocorrido neste caso relatado. O trabalho em questão ressalta ainda a necessidade de uma criteriosa análise histopatológica para o diagnóstico deste tumor, a fim de diferenciá-lo de outras neoplasias, como o adenoma pleomórfico e o carcinoma adenóide cístico, pois ambos possuem terapêuticas e prognósticos distintos.

Sugerimos para trabalhos futuros uma análise clínica e histopatológica criteriosa, para que o diagnóstico seja correto , e o paciente seja encaminhado ao serviço especializado o mais breve possível, melhorando assim o prognóstico para ele.

\section{Referências}

Abu El-Naaj, I., Leiser, Y., Wolff, A., Peled, M. (2011). Polymorphous low grade adenocarcinoma: case series and review of surgical management. J Oral Maxillofac Surgery, 69, 1967-72.

Araujo, V., C., Passador-Santos, F., Turssi, C., Soares, A., B., Araujo, N., S. (2013). Polymorphous low-grade adenocarcinoma: an analysis of epidemiological studies and hints or pathologists. Diagn Pathol, 6, 1-8.

Barnes, L., Everson, J., W., Reichart, P., Sidransky, D. (2005). Wased Health Organization Classification of Tumors. Pathology and genetics of head and neck tumors. International Agency for research on cancer.

Baroni, Júnior, D., Liutti, V., T., Gama, R., R. (2008). Estudo retrospectivo de pacientes portadores de neoplasias de glândulas salivares: levantamento de 75 casos. Rev Med Res, 10(1), 16-24.

Buchner, A., Merrell., P., W., Carpenter, W., M. (2007). Relative frequency of intra-oral minor salivary gland tumors: a study of 380 cases from northern California and comparison to reports from other parts of the world. J Oral Pathol Med, 36, 207-14.

B., W., Damm, D., D., Allen, C., M., Bouquot, J., E. (2009). Patologia oral e maxilofacial. $3^{\text {a }}$ ed. Rio de Janeiro, Elsevier.

Carboni, S., S., C., M. (2011). Padrão de expressão das gtpases RHO em adenoma pleomórfico de parótida. Universidade Federal do Triangulo Mineiro.

Fava, A., S., (2001). Diagnóstico, estadiamento e tratamento cirúrgico dos tumores malignos das glândulas salivares. Tratado de Cirurgia de Cabeça e Pescoço e Otorrinolaringologia. São Paulo, Atheneu (pp.791-812). 
Research, Society and Development, v. 10, n. 9, e59910918461, 2021

(CC BY 4.0) | ISSN 2525-3409 | DOI: http://dx.doi.org/10.33448/rsd-v10i9.18461

Fife, T., A., Smith, B., Sullivan, C., A., Browne, J., D., Waltonen, J., D. (2013) Polymorphous low-grade adenocarcinoma: a 17 patient case series. Am J Otolaryngol, 34, 445-8.

J., Á., Ciulba, J., J., Jordan, R., C., K. (2008). Patologia Oral, correções clínico patológicas. $5^{\text {a }}$ Ed. Saunders Elsevier, Rio de Janeiro.

Jones, A., V., Craig, G., T., Speight, P., M., Franklin, C., D. (2008). The range and demographics of salivary gland tumors diagnosed in a UK population. Oral Oncol, 44(4), 407-17.

J., T., Thompson, L., D., R., Frommelt, R., A., Wenrig, B., M., Kessler, H., P., (1999). Polymorphous Low - Grad Adenocarcinoma a clinicopathologic study of 164 cases. American cancer society, 86(2), 207-219.

Lima, S., S., Soares, A., F., Amorim, R., F., B., Freitas, R., A. (2005). Perfil epidemiológico das neoplasias de glândulas salivares: análise de 245 casos. Rev Bras Otorrinolaringol, 71(3), 335-40.

Loiola, R., S., Matos, F., R., Nonaka, C., F., W., Lopes, F., F., Cruz, M., C., F., N. (2009). Perfil epidemiológico das neoplasias de glândulas salivares diagnosticadas em São Luís-MA. J Bras Patol Med Lab, 45(5), 413-20.

M., A., Wenig, B., M. (2009). Classificação de Tumores da Organização Mundial de Saúde. Genética e Patologia dos Tumores de Cabeça e de Pescoço. Ed. Santos. Gen/Grupo Editorial Nacional.

Mc., Hugh, Jonathan, B., M., D., V., Daniel, W., M., D., Barnes, E., Leon, M., D. (2009). Update on Selected Salivary Gland Neoplasms. Archives of Pathology e Laboratory Medicine, 1763-74.

M., C., Penna, K., Vinaski, M. (2000). Polymorphous Low - Grade Adenocarcinoma of the oral cavity. New York State Dental Journal, 28-32.

Milsa, Sarmento, D., J., S., Silveira, E., J., D., Oliveira, I., C., P., Costa, A., L., L. (2010). Adenocarcionoma polimorfo de baixo grau: relato de um caso adicional e uma atualização dos principais aspectos sobre seu diagnóstico. Rev. Odontol UNESP, 311-315.

M., S., Sabri, A., N., Lewis, J., E., Olsen, K., D. (2006). Retrospective study and review of polymorphous low-grade adenocarcinoma. Laryngoscope, 116, 2145-9.

Neville, B., W., Damm, D., D., Allen, C., M., Bouquot, J., E. (2008). Patologia oral \& maxillofacial. $2^{\mathrm{a}}$ Ed. Guanabara Koogan, Rio de Janeiro.

O’ Rourke, A., K., Gourin, C., G., Wade, Z., K., Hessler, R., B. (2006). Polymorphous Low - Grade Adenocarcinoma of the parotid gland. Ear, Nose e Thoat Journal, 669-71.

S., Kumar, C., A., Raghav, N. (2011). Polymorphous Low- Grade Adenocarcinoma of the Palate: Report of a case and Review of Literature. International journal of Head and Nick Surgery, 57-60.

Seethala, R., R., Johnson, J., T., Barnes, E., L., Myers, E., N. (2010). Polymorphous low-grade adenocarcinoma: the University of Pittsburgh experience. Arch Otolaryngol Head Neck Surg, 136, 385-92.

Soames, J., V., Southam, J., C. (2008). Patologia Oral. 4 Ed. Editora Gen/Guanabara Koogan, Rio de Janeiro.

Toida, M., Shimokawa, K., Makita, H., Kato, K., Kobayashi, A., Kusunoki, Y. (2005). Intraoral minor salivary gland tumors: a clinicopathological study of 82 cases. Int J Oral Maxillofac Surg, 34, 528-32. 